Research Paper

\title{
Is liver involvement overestimated in COVID-19 patients? A meta-analysis
}

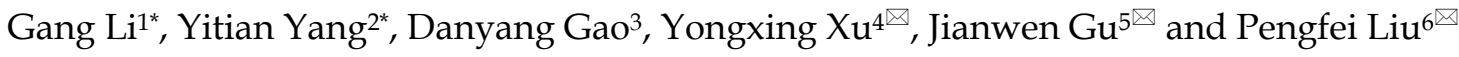 \\ 1. Department of General Surgery, Peking University Third Hospital, Haidian District, Beijing 100191, China. \\ 2. Department of Anesthesiology and Perioperative Medicine, Henan Provincial People's Hospital, People's Hospital of Zhengzhou University, Zhengzhou, \\ 450003, China. \\ 3. Department of Anesthesiology, Capital Medical University affiliated Beijing Shijitan Hospital, Beijing 100038, China. \\ 4. Department of Nephrology, PLA Strategic Support Force Characteristic Medical Center, Beijing 100101, China. \\ 5. The Leading Group on COVID-19 Prevention and Control, People's Liberation Army Strategic Support Force Characteristic Medical Center, Beijing 100101, \\ China. \\ 6. Department of Anesthesiology, Capital Medical University affiliated Beijing Shijitan Hospital, Beijing 100038, China.
}

*These authors contribute equally to this work.

$\square$ Corresponding authors: Xu Yongxing, Gu Jianwen \& Pengfei Liu contributed equally to this work. Yongxing Xu, MD. Department of Nephrology, PLA Strategic Support Force Characteristic Medical Center, Beijing 100101, China. E-mail: xuyongxingstar@163.com. Tel: +86- 01066356729. Jianwen Gu, MD. The Leading Group on COVID-19 Prevention and Control, People's Liberation Army Strategic Support Force Characteristic Medical Center, Beijing 100101, China. E-mail: gujianwen5000@sina.com. Tel: +86-01066356729. Pengfei Liu, MD. Department of Anesthesiology, Beijing Shijitan Hospital, Capital Medical University, No. 10 Tieyi Road, Yangfangdian, Haidian District, Beijing 100038, China. Email: sfflpf@126.com. Tel: +86-01063926507.

(c) The author(s). This is an open access article distributed under the terms of the Creative Commons Attribution License (https://creativecommons.org/licenses/by/4.0/). See http://ivyspring.com/terms for full terms and conditions.

Received: 2020.07.27; Accepted: 2020.12.02; Published: 2021.01.18

\begin{abstract}
Background: Considering transaminase more than the upper limit of normal value as liver injury might overestimate the prevalence of liver involvement in COVID-19 patients. No meta-analysis has explored the impact of varied definitions of liver injury on the reported prevalence of liver injury. Moreover, few studies reported the extent of hypertransaminasemia stratified by COVID-19 disease severity.

Methods: A literature search was conducted using PubMed and Embase. The pooled prevalence of liver injury and hypertransaminasemia was estimated.

Results: In total, 60 studies were included. The overall prevalence of liver injury was $25 \%$. Compared to subgroups with the non-strict definition of liver injury (33\%) and subgroups without giving detailed definition (26\%), the subgroup with a strict definition had a much lower prevalence of liver injury (9\%). The overall prevalence of alanine aminotransferase (ALT) and aspartate aminotransferase (AST) elevation was $19 \%$ and $22 \%$. The prevalence of elevated ALT and AST were significantly higher in severe COVID-19 cases compare to non-severe cases (31\% vs $16 \%$ and $44 \%$ vs $11 \%$ ). In critically ill and fatal cases, no difference was found in the prevalence of elevated ALT (24\% vs $30 \%$ ) or AST (54\% vs $49 \%$ ). Sensitivity analyses indicated that the adjusted prevalence of ALT elevation, AST elevation, and liver injury decreased to $14 \%, 7 \%$, and $12 \%$.

Conclusion: The overall prevalence of liver injury and hypertransaminasemia in COVID-19 patients might be overestimated. Only a small fraction of COVID-19 patients have clinically significant liver injury. The prevalence of hypertransaminasemia was significantly higher in severe COVID-19 cases compare to non-severe cases. Hence, in severe COVID-19 patients, more attention should be paid to liver function tests.
\end{abstract}

Key words: COVID-19; hypertransaminemia; liver injury; alanine aminotransferase; aspartate aminotransferase; meta-analysis

\section{Introduction}

The novel severe acute respiratory syndrome coronavirus 2 (SARS-CoV-2) virus, which causes coronavirus disease 2019 (COVID-19), was discovered in China in December 2019 and quickly spread into an ongoing global pandemic [1, 2]. COVID-19 has continued to spread for the greater part of a year and 
the second wave of COVID-19 has been under way around the world [3].

A growing body of research has reported that some patients with COVID-19 have abnormal liver function tests and liver injury [4-6]. There are already several systematic reviews and meta-analyses addressing this topic [7-9]. However, few of these studies have reported the pooled prevalence of transaminase elevation and liver injury, and when reported, the results have been inconsistent [10,11].

The clinical manifestation of COVID-19 ranges from mild to critically ill and fatal cases. Patients with severe COVID-19 have a higher risk of liver involvement [11]. To date, there is a gap in knowledge regarding the extent of liver injury and increased transaminase levels stratified by COVID-19 disease severity. Moreover, we think it is not rigorous that some investigators considered the transaminase more than the upper limit of normal value as liver injury [11]. In addition, because elevated transaminase might be due to myocardial injury or muscle injury induced by SARS-CoV-2 infection [12, 13], the prevalence of liver injury might be overestimated. There is a lack of a uniform definition of acute liver injury caused by COVID-19. One previous study defined liver injury in COVID-19 as an ALT or AST level greater than 3 times the upper limit of normal [14]. By contrast, one study indicated that liver injury was diagnosed according to the elevation of bilirubin and aminotransferase [15]. The definitions of liver injury have a major impact on the results of pooled analysis, however, no meta-analysis explored this.

Therefore, we conducted this meta-analysis of clinical studies on the COVID-19 to comprehensively explore the prevalence of transaminase elevation and liver injury in COVID-19 patients. We analyzed the data based on the severity of the COVID-19 to further discuss the liver involvement in different populations. More importantly, based on the different definitions of liver injury, we conducted subgroup analysis to explored the impact of varied definitions of liver injury on the prevalence of liver injury.

\section{Methods}

This meta-analysis followed the preferred reporting items for systematic reviews and meta-analyses (PRISMA) statement (Supplementary Table 1) [16] and was registered with the International Prospective Register of Systematic Reviews (PROSPERO) and assigned registration number CRD42019120201. Moreover, the protocol of this meta-analysis has been published [9].

\section{Search strategy and selection criteria}

We searched PubMed and Embase electronic databases on April 13, 2020, for potentially relevant studies from December 2019 using relevant words and medical subject headings as follows: severe acute respiratory syndrome coronavirus 2, SARS-CoV-2, sars cov 2, 2019-nCoV, 2019 novel CoV, 2019 novel coronavirus, coronavirus disease 19, coronavirus disease 2019, COVID-19, COVID-2019, and novel coronavirus-infected pneumonia (Supplementary Table 2). The reference list of the eligible articles and relevant reviews were manually searched to identify additional studies. There were no language restrictions.

Two independent reviewers (PL and GL) assessed the eligibility of studies with a standardized approach. Discrepancies were resolved by discussion with a third individual (YX). Studies in languages other than English or Chinese were translated into English using Google Translate. When there were multiple studies from the same cohort, the study with the largest sample size was used. If the sample size did not vary, the most recent paper was used.

Two authors (PL and GL) independently extracted data into a standardised excel spreadsheet, including study characteristics, prevalence and definition of acute liver injury, and prevalence of elevated alanine aminotransferase (ALT) and aspartate aminotransferase (AST) in patients with COVID-19. Data on the impact of acute liver injury on the clinical course of COVID-19 disease and prognosis were collected if available. Published observational studies reporting the prevalence of acute liver injury and/or hypertransaminasemia were included. Single case studies and studies with less than 10 participants were excluded. Although we were not interested in interventions, we included nonrandomized analyses performed in populations derived from randomised controlled trials.

\section{Assessing the risk of bias}

The risk of bias of the included studies was assessed independently by two reviewers (PL and GL) using the Joanna Briggs Institute's critical appraisal checklist [17]. Any disagreement was discussed by consultation or resolved by a third investigator $(\mathrm{YX})$.

\section{Outcomes and definitions}

The prevalence of acute liver injury in patients with COVID-19 was the main outcome. The prevalence of elevated ALT and AST in patients with COVID-19 were secondary outcomes. Acute liver injury and elevated ALT and AST were defined based on criteria used in the individual studies. Based on previous studies,[14, 18-20] we adopted a relatively strict definition of acute liver injury as ALT and/or 
AST higher than 3-fold of the upper limit of normal (ULN), or alkaline phosphatase and/or total bilirubin (TBIL) higher than 2-fold of the ULN. In studies that considered any ALT, AST, alkaline phosphatase, and/ or TBIL level higher than the ULN as liver injury, the definition was considered not strict or insufficiently rigorous. As in a previous study,[11] disease severity of COVID-19 was defined based on criteria used in the individual studies; meanwhile, COVID-19 patients requiring admission to the intensive care unit or with acute respiratory distress syndrome were classified as severe cases [11]. Fatal cases were also considered as severe cases.

\section{Statistical analysis}

The pooled prevalence of hypertransaminasemia and acute liver injury was estimated using a random-effects model [21]. Forest plots were generated to display prevalence with corresponding 95\% confidence intervals (CIs). The heterogeneity between studies was investigated statistically using the chi-square test and $\mathrm{I}^{2}$ statistic. $\mathrm{I}^{2}$ values of $25 \%$, $50 \%$, and $75 \%$ corresponded to low, medium, and high levels of heterogeneity, respectively [22]. Subgroup analyses were undertaken based on the severity of COVID-19 and age (adult vs children). The impact of the various definitions of liver injury on the reported prevalence of liver injury was also explored. Sensitivity analyses were performed using the Duval and Tweedie nonparametric "trim and fill" procedure $[23,24]$. Funnel plots, Egger's regression asymmetry test, and Begg's test were used to evaluate publication bias [25, 26]. A two-sided $p$-value $<0.05$ was considered significant for all analyses. All analyses were performed using Stata software v 15.0 (StataCorp, College Station, TX, USA).

\section{Results}

Initially, 6193 publications were identified, 1802 of which were duplicates and excluded. Subsequently, 4328 articles were excluded after browsing the titles, abstracts, and full-text reviews for relevancy. Finally, a total of 60 [1, 4-6, 12, 14, 15, 27-79] articles that met the inclusion criteria were included in the study (Fig. 1).

The main characteristics of patients and studies included in the meta-analysis were shown in Supplementary Table 3. The risk of bias of the included studies was shown in Supplementary Table 4.
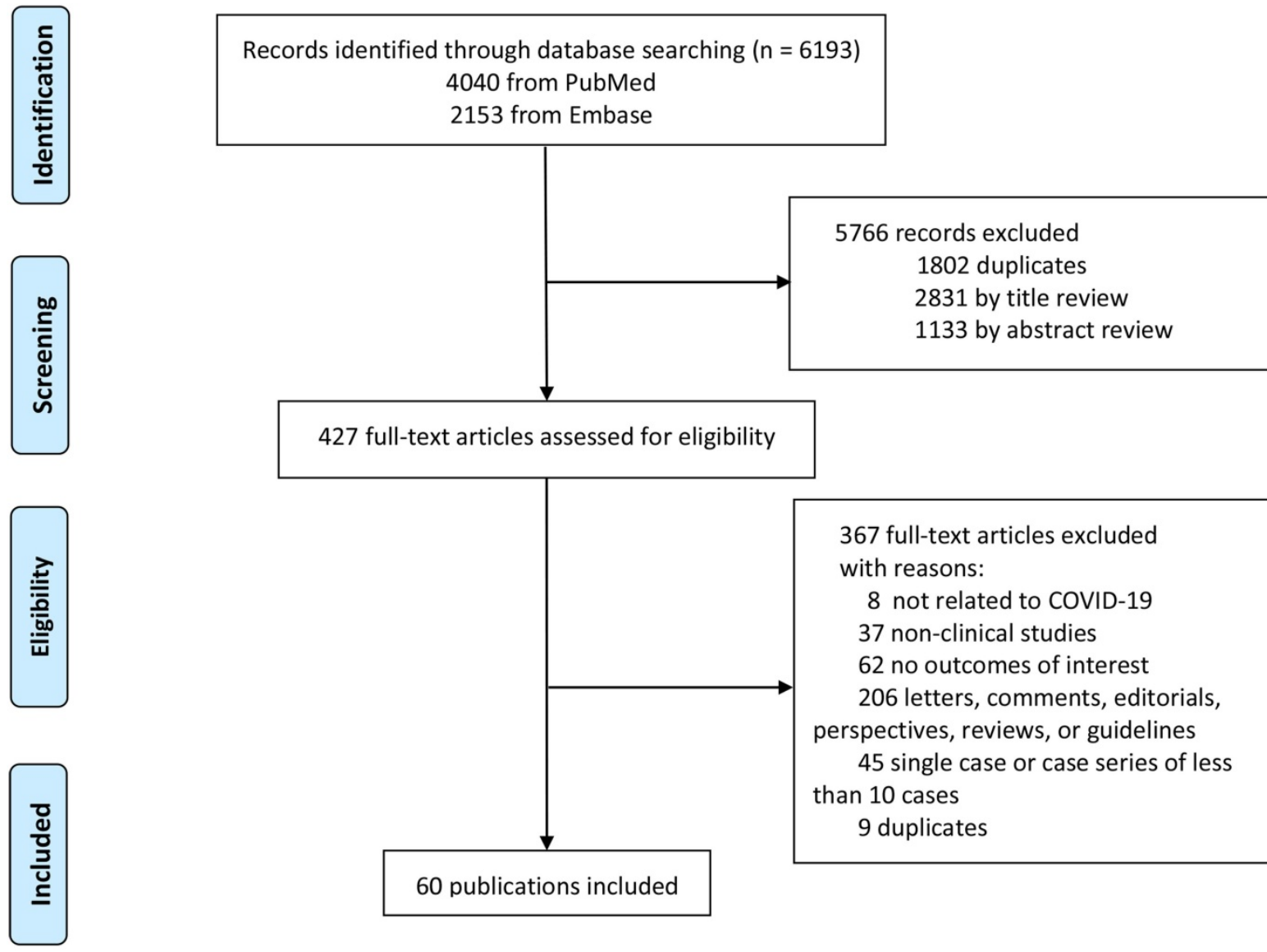

60 publications included 


\begin{tabular}{|c|c|c|c|}
\hline Study & Liver injury & Total & Incidence $(95 \% \mathrm{CI})$ \\
\hline \multicolumn{3}{|l|}{ Strict } & \\
\hline Arentz, M. ${ }^{14}$ & 3 & 21 & $0.14(0.03,0.36)$ \\
\hline Cai, Q. ${ }^{29}$ & 44 & 298 & $0.15(0.11,0.19)$ \\
\hline Chen, G. ${ }^{79}$ & 1 & 21 & $0.05(0.00,0.24)$ \\
\hline Chen, $\mathrm{Tao}^{78}$ & 13 & 274 & $0.05(0.03,0.08)$ \\
\hline \multirow[t]{2}{*}{ Subtotal } & & & $0.09(0.03,0.17)$ \\
\hline & & & \\
\hline \multicolumn{3}{|l|}{ Not reported } & \\
\hline $\mathrm{Du}, \mathrm{Y} .{ }^{36}$ & 30 & 85 & $0.35(0.25,0.46)$ \\
\hline Guo, T. ${ }^{38}$ & 19 & 187 & $0.10(0.06,0.15)$ \\
\hline Tu, W. J. ${ }^{59}$ & 45 & 174 & $0.26(0.20,0.33)$ \\
\hline Yang, X. ${ }^{69}$ & 15 & 52 & $0.29(0.17,0.43)$ \\
\hline Zha, L. ${ }^{71}$ & 12 & 31 & $0.39(0.22,0.58)$ \\
\hline \multirow{2}{*}{\multicolumn{3}{|c|}{ Subtotal }} & $0.26(0.15,0.39)$ \\
\hline & & & \\
\hline \multicolumn{3}{|l|}{ Non-strict } & \\
\hline Ji, D. ${ }^{4}$ & 152 & 202 & $0.75(0.69,0.81)$ \\
\hline Lian, J. ${ }^{48}$ & 82 & 788 & $0.10(0.08,0.13)$ \\
\hline Lin, L. ${ }^{49}$ & 31 & 95 & $0.33(0.23,0.43)$ \\
\hline Wang, F. ${ }^{62}$ & 15 & 52 & $0.29(0.17,0.43)$ \\
\hline Wang, L. ${ }^{63}$ & 4 & 16 & $0.25(0.07,0.52)$ \\
\hline Wang, Y. ${ }^{15}$ & 54 & 344 & $0.16(0.12,0.20)$ \\
\hline Yao, N. ${ }^{70}$ & 22 & 40 & $0.55(0.38,0.71)$ \\
\hline \multirow{2}{*}{\multicolumn{3}{|c|}{ Subtotal }} & $0.33(0.14,0.56)$ \\
\hline & & & \\
\hline \multicolumn{3}{|c|}{ Heterogeneity between groups: $p=0.015$} & \\
\hline \multirow{2}{*}{\multicolumn{3}{|c|}{ Overall $\left(\mathrm{I}^{\wedge} 2=96.85 \%, \mathrm{p}=0.00\right)$}} & $0.25(0.15,0.35)$ \\
\hline & & & \\
\hline
\end{tabular}

Figure 2. Estimated incidence prevalence of liver injury based on definition.

From 16 studies $[14,15,29,36,38,44,48,49,59$, $62,63,69-71,78,79]$, the overall prevalence of liver injury in the COVID-19 patients was $25 \%$ (95\% CI: $0.15,0.35)$ with significant heterogeneity among the studies $\left(I^{2}=96.85 \%\right)$, as shown in Figure 2 and Supplementary Table 5. The definition of liver injury varied among the studies. Four studies were considered to have strict definitions [14, 29, 78, 79], seven were considered to have non-strict definitions $[15,44,48,49,62,63,70]$, and five did not provide definitions [36, 38, 59, 69, 71]. Subgroup analysis showed a significant difference in the prevalence of liver injury among the three subgroups according to different type of definition ( $p=0.015$; Figure 2$)$. The prevalence of liver injury in subgroups with strict, non-strict, and unreported definitions were 9\% $(95 \%$ CI: $0.03,0.17), 33 \%$ (95\% CI: 0.14, 0.56), and 26\% (95\% CI: $0.15,0.39$ ), respectively (Figure 2). In subgroup with strict definition, the prevalence of liver injury was $17 \%(95 \%$ CI: $0.04,0.34)$ in patients with severe COVID-19, with significant heterogeneity among the studies $\left(I^{2}=83.36 \%\right.$; Supplementary Figure 1$)$.

Data from 42 studies [4-6, 12, 27-37, 39-46, 49, 50, $53-57,64-68,72-78]$ were pooled for meta-analysis of elevated ALT levels in COVID-19 patients, resulting in prevalence of $19 \%$ (95\% CI: 0.16, 0.23; Figure 3). Significant heterogeneity among the studies was detected $\left(I^{2}=88.04 \% ; p<0.001\right)$. Subgroup analyses revealed that the prevalence of elevated ALT in patients with severe COVID-19 was 30\% (95\% CI: $0.24,0.38)$, which was significantly higher than that in patients with non-severe cases $(15 \%$; $95 \%$ CI: 0.07, $0.25)$ ( $p=0.011$ for subgroup difference; Figure 4). However, there was no significant difference in the prevalence of elevated ALT levels between critically ill patients $(24 \%$; 95\% CI: 0.10, 0.41) and fatal cases $(30 \%$; 95\% CI: $0.20,0.41) \quad(p=0.611$ for subgroup difference; Supplementary Figure 2). 


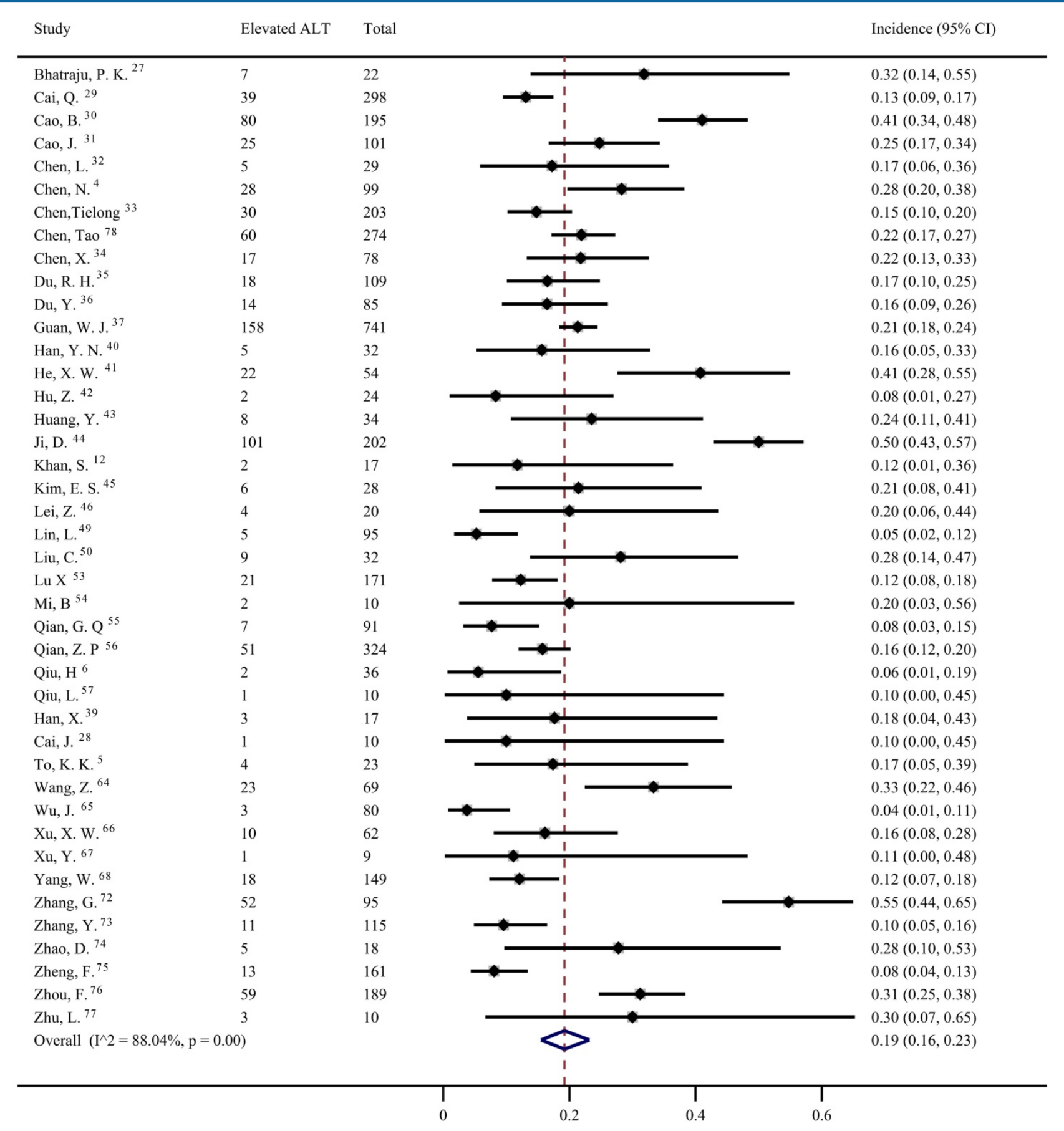

Figure 3. The pooled estimate of elevated alanine aminotransferase in patients with COVID-19.

Forty studies $[1,4,6,12,27-30,32-37,39-44,46$, $49,50,53-58,60,64,65,67,68,72-75,78,79]$ were included to evaluate the prevalence of elevated AST in COVID-19 patients. The pooled prevalence was $22 \%$ (95\% CI: 0.18, 0.27; Figure 5) with significant heterogeneity noted among the studies $\left(I^{2}=89.55 \%\right.$; $p<$ 0.001). Subgroup analyses showed that the patients with severe COVID-19 had a significantly higher risk of elevated AST than non-severe COVID-19 patients (44\%; 95\% CI: 0.36 , 0.5 vs $11 \%$; 95\% CI: $0.05,0.18)$ ( $p<$ 0.001 for subgroup difference; Figure 6). However, there was no significant difference in the prevalence of elevated AST between the critically ill patients (54\%; 95\% CI: $0.37,0.72$ ) and the fatal cases $(49 \%$; $95 \%$ CI: $0.36,0.61)(p=0.601$ for subgroup difference; Supplementary Figure 3).

Four studies [47, 51, 52, 61] reported the prevalence of elevated transaminase (a mixture of data on elevated ALT or AST), but neither exact number of elevated ALT nor AST was available in these studies. Hence, we presented a narrative synthesis. These four studies came from China and included relatively fewer subjects (ranging from 10 to 31. Only two studies [51, 52] reported the normal 
range of ALT and AST. The prevalence of elevated transaminase ranged from $20 \%$ to $48 \%$.

The overall prevalence of elevated ALT in paediatric patients was significantly lower than that in adult patients $(10 \%$ [95\% CI: $6-14]$ vs $21 \%$ [95\% CI 13-30]; $p=0.032$ for subgroup difference; Supplementary Figure 4). The prevalence of elevated AST in paediatric patients was also lower than that in adult patients, however, the difference did not reach significance $(14 \%$ [ $95 \%$ CI: $7-22$ ] vs $24 \%$ [95\% CI: 16-33]; $\quad p=0.094$ for subgroup difference; Supplementary Figure 5). We further compared the prevalence of elevated ALT and AST in pediatric patients to severe adult and non-severe adult COVID-19 patients by subgroup analysis (Supplementary Figure 6 and 7). The prevalence of elevated ALT in paediatric patients and adults with severe and non-severe COVID-19 were 10\% (95\% CI: 6, 14\%), 27\% (95\% CI: 16, 39\%), and 6\% (95\% CI: 3, $12 \%)$, respectively and the prevalence of elevated AST were $14 \%$ (95\% CI: 7, 22\%), 39\% (95\% CI: 20, 59\%), and $8 \% \quad(95 \%$ CI: 4, 14\%), respectively. All of the differences in elevated ALT and AST among the subgroups were significant $(p<0.001$ and $p=0.001$ for subgroup difference, respectively). We found that no study reported the prevalence of liver injury in children with COVID-19.

Although the funnel plot of ALT was slightly asymmetric (Supplementary Figure 8), neither the Begg's nor Egger's tests were statistically significant ( $p=0.111$ and 0.080 , respectively). The funnel plot of AST displayed a clear asymmetry (Supplementary Figure 9). The Begg's test of AST results showed no statistical significance ( $p=0.907)$, but the Egger's test showed statistical significance $(p<0.001)$. Through visual inspection, the funnel plots of liver injury showed obvious asymmetry (Supplementary Figure 10). The Egger's test showed statistical significance ( $p$ $=0.045)$ but the Begg's test was not statistically significant $(p=0.126)$.

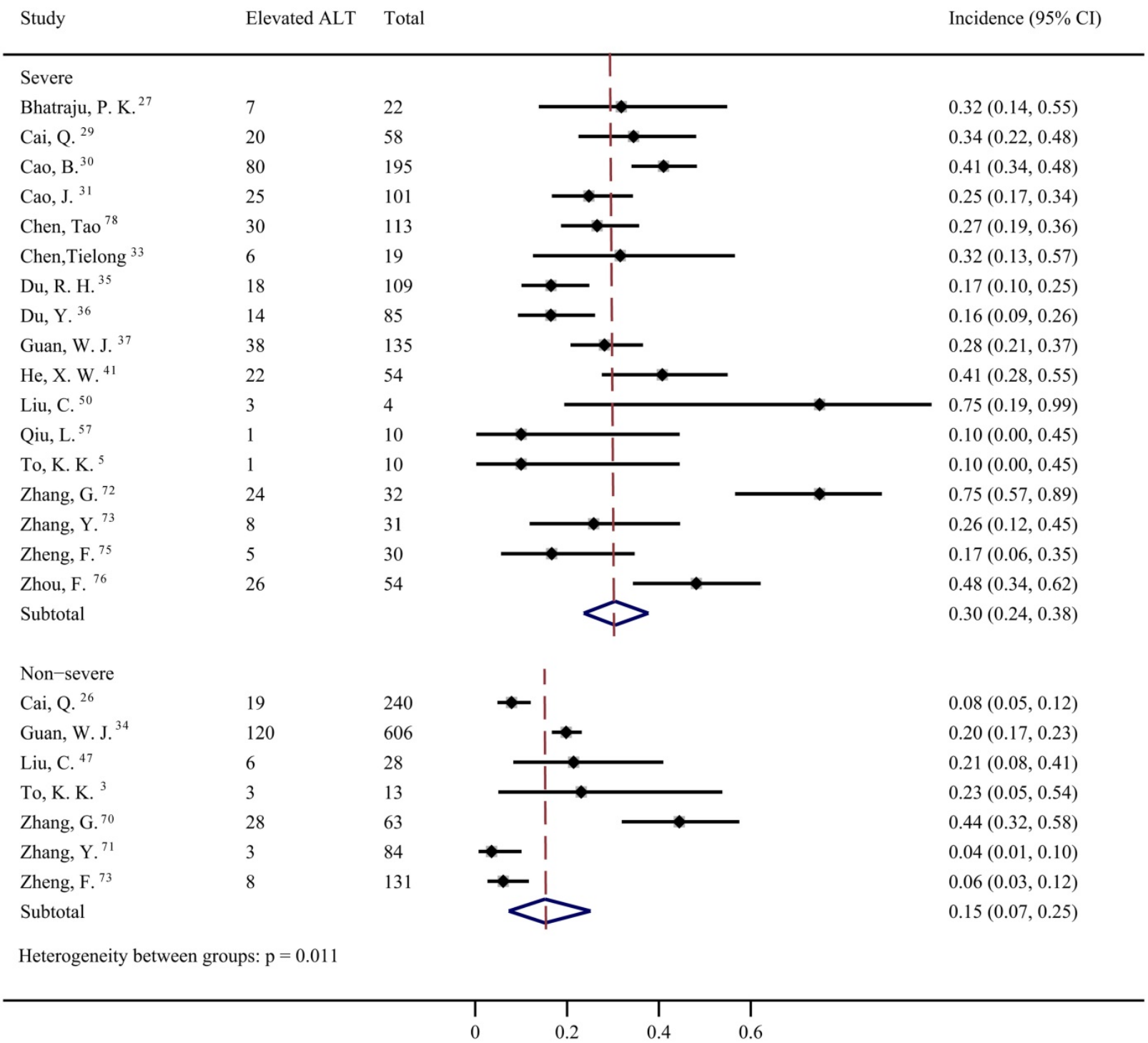

Figure 4. Pooled estimate of elevated alanine aminotransferase in severe and non-severe patients with COVID-19. 


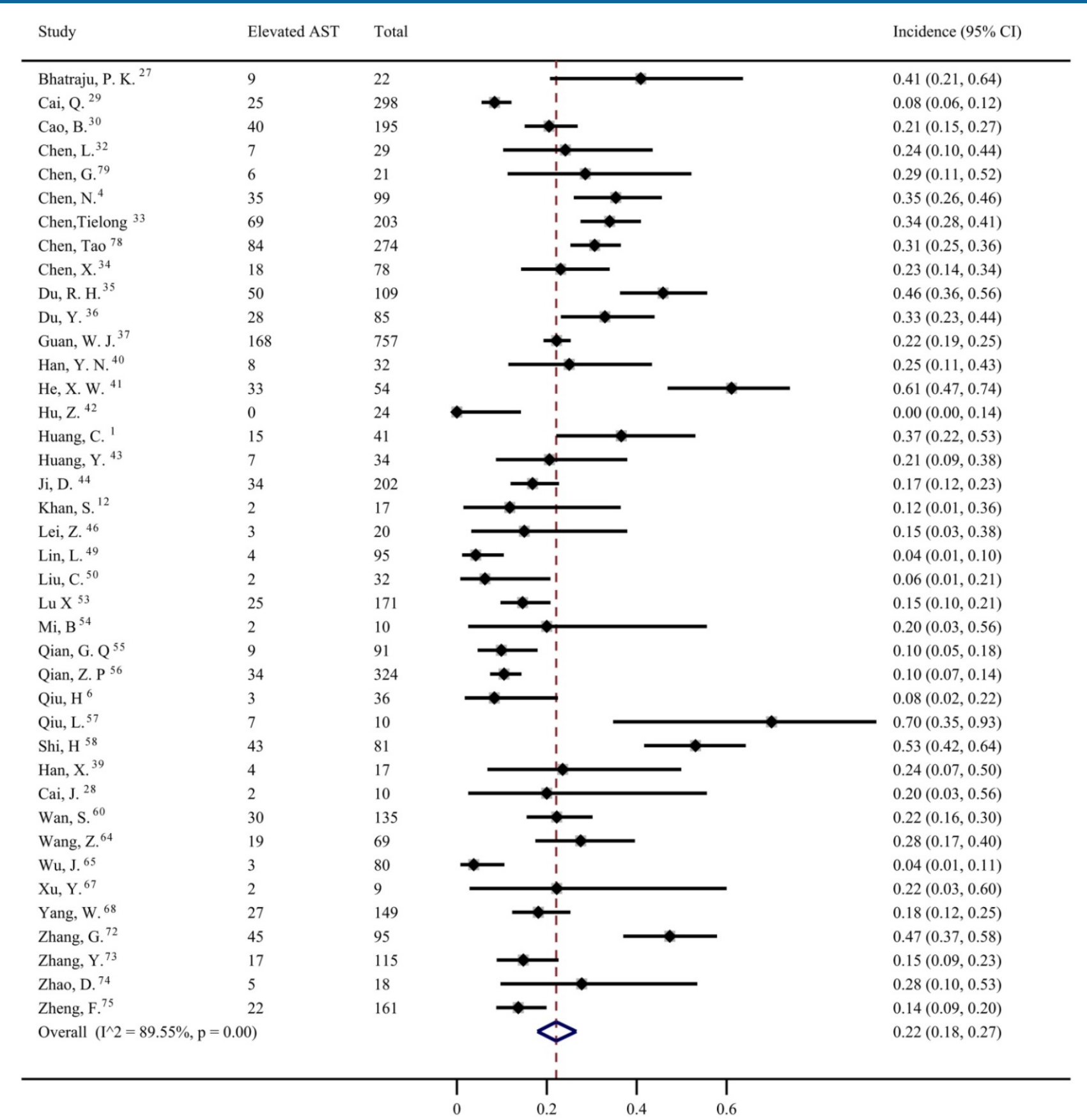

Figure 5. The pooled estimate of elevated aspartate aminotransferase in patients with COVID-19.

Subsequently, we performed sensitivity analyses by the Duval and Tweedie nonparametric "trim and fill" procedure. As a result of the ALT sensitivity analysis, 13 studies were deemed as missing and were imputed into the trim and fill plot (Supplementary Figure 11). The adjusted point estimate decreased to 14\% (95\% CI: $10 \%-17 \%)$ under the random effects model. For AST, 20 studies were missing, which were entered using the funnel plot (Supplementary Figure 12). After adjusting, the point estimate was reduced to $7 \%$ (95\% CI: 3\%-12\%) under the random effects model. For liver injury, after adding 7 studies into the plot, the adjusted point estimate was reduced to $12 \%$
(95\% CI: 3\%-21\%) under the random effects model (Supplementary Figure 13).

One study conducted by Yao [70] indicated that there were more severe cases in COVID-19 patients with liver injury compared to those without liver injury $(77.3 \%$ vs $27.8 \%)$. Among the studies included in the present meta-analysis, three discussed the association between elevated transaminase and the prognosis of patients. Chen [33] reported that the patients with elevated AST (AST $>40 \mathrm{U} / \mathrm{L}$ ) tended to have a higher risk of death but without a significant difference (odds ratio: 2.04; 95\% CI: 0.56, 7.38; $p$ $=0.28$ ). Zhou [76], in a retrospective study, found that 


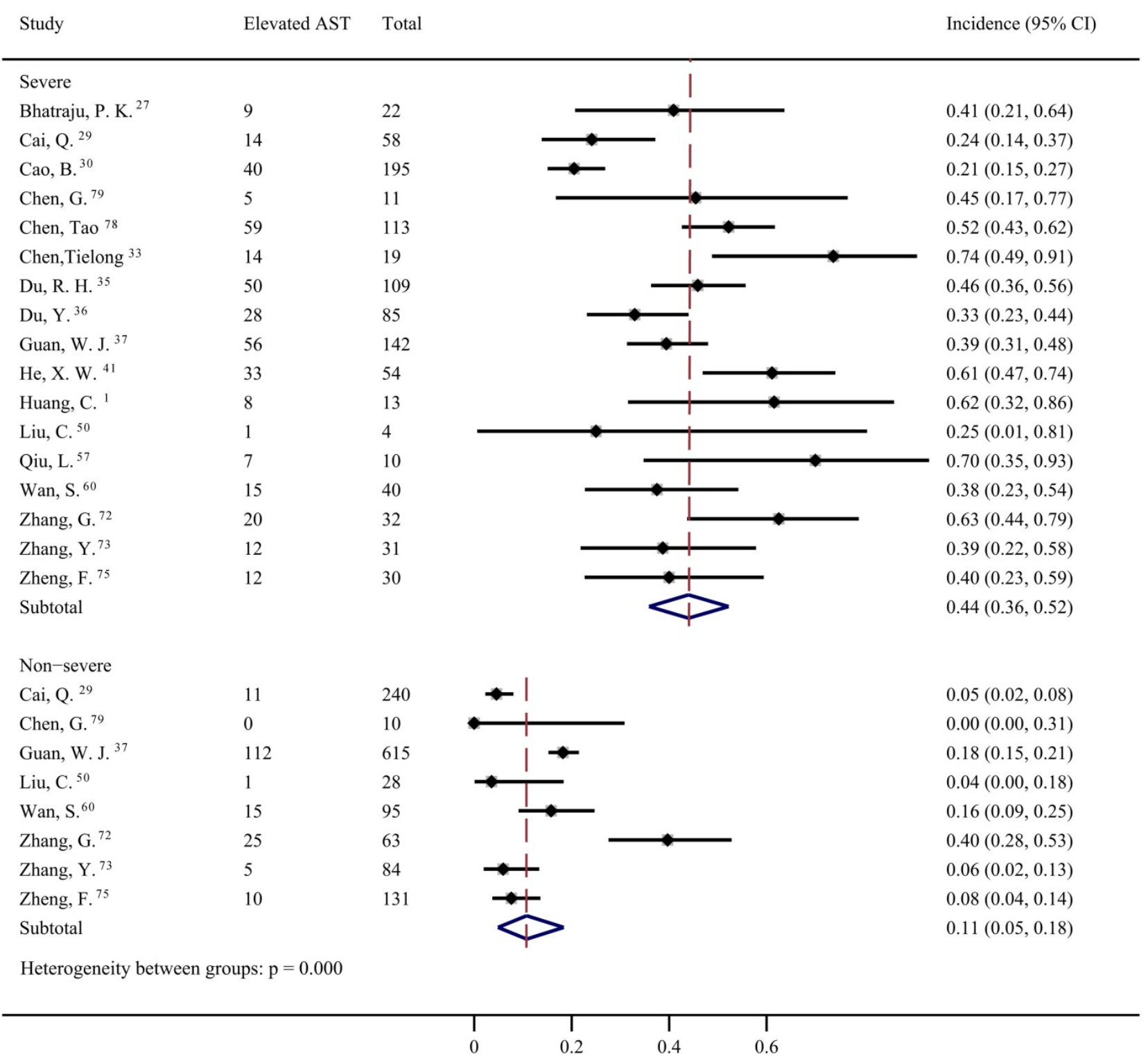

Figure 6. Pooled estimate of elevated aspartate aminotransferase in severe and non-severe patients with COVID-19.

the odds of death were higher in patients with elevated ALT (ALT $>40 \mathrm{U} / \mathrm{L}$ ) according to univariable analysis (odds ratio: 2.87; 95\% CI: 1.48, 5.57; $p<0.01$ ), while elevated ALT (ALT > $40 \mathrm{U} / \mathrm{L}$ ) was not associated with an increased risk of death by further multivariate regression analysis $(p>0.05)$. However, Zhang et al. [72] found that both higher ALT and AST activity were related to the composite endpoint (including mechanical ventilation, admission to the intensive care unit, and death) $(p<0.05)$.

\section{Discussion}

In this comprehensive meta-analysis, the overall prevalence of liver injury defined by the criteria used in the individual studies was $25 \%$. By subgroup analysis, we found the various definitions of liver injury had a significant impact on the reported prevalence of liver injury. The prevalence of clinically significant liver injury defined by strict criteria was only $9 \%$. Sensitivity analyses indicated that the overall prevalence of liver injury and increased aminotransferase might be overestimated.

The underlying mechanisms of COVID-19related liver injury remain unclear. Clinically, liver injury might be associated with pre-existing liver diseases, viral infection, drug toxicity, and systemic inflammation [80-84]. Pneumonia-associated hypoxia might also contribute to liver injury [84]. Some studies report that the angiotensin-converting enzyme 2 (ACE 2 ), the receptor of SARS-CoV-2, mainly located in the lungs, is also widely expressed in liver cells [80]. Besides, pulmonary hypertension and reduced right heart function can be observed in COVID-19 patients [85-87]. Acute heart failure was one of the most 
common critical complications during the exacerbation of COVID-19 [78]. Liver has a high metabolic activity and perfusion rate. Liver injury might be brought about by acute circulatory changes, in the setting of which the liver's compensatory mechanism is being insufficient [88]. Hence, liver injury in COVID-19 patients might also be related to haemodynamic issues.

Currently, there is no uniform definition of liver injury for COVID-19 patients. Several studies considered the abnormal liver function tests in the COVID-19 patients as the liver injury [11, 15], but this is not a sufficient criterion because mild aminotransferase elevation could also occur in COVID-19 patients with myocardial damage and/or muscle injury $[12,13]$. By contrast, one study defined liver injury in COVID-19 as an ALT or AST level greater than 3 times the ULN [14]. Hence, based on previous studies and definitions of other types of liver injury (such as drug-induced liver injury, liver injury in HIV, and liver injury in adult dengue infection) [14, 18-20], we adopted a relatively strict definition of acute liver injury, which was ALT and/or AST higher than 3-fold of the ULN, or alkaline phosphatase and/or TBIL higher than 2-fold of the ULN [9].

The overall prevalence of liver injury based on the definition included in the individual studies was $26 \%$, higher than the $19 \%$ reported by Mao et al.[11] Besides, our results suggested that the definition of liver injury has a major impact on the reported prevalence in patients with COVID-19. According to the stricter definition, we found the prevalence of liver injury was only $9 \%$.

Although previous meta-analyses have addressed abnormal liver function $[7,8,10,11,74$, 89-92], few studies have investigated the prevalence of elevated aminotransferase [10, 11]. Several previous meta-analyses [7, 8, 89], estimated weighted mean difference or standardised mean difference of liver function measure in COVID-19 patients, but not reported the prevalence of elevated aminotransferase. Mao et al. indicated that the pooled prevalence of elevated ALT was 18\% from 14 studies and elevated AST was $21 \%$ by combining 14 studies [11]. Another study reported that the pooled prevalence estimates of elevated ALT and elevated AST were both 15.0\% [10]. In this meta-analysis, 17 and 16 studies were included when performing the pooled prevalence of ALT and AST, respectively [10]. In the present meta-analysis, the overall prevalence of elevated ALT and AST was $19 \%$ and $22 \%$, respectively. These results are similar to those in the study conducted by Mao et al. [11]. However, the present study included more studies. Specifically, there were a total of 42 studies that reported the prevalence of elevated ALT and 40 studies that reported the prevalence of elevated AST. However, abnormal ALT and AST level in COVID-19 patients need to be interpreted cautiously $[93,94]$. As we stated, not all abnormal liver function tests mean that patients with COVID-19 have liver injury, and elevated aminotransferases might partly result from myocardial injury and muscle injury [94].

There is limited data from meta-analyses on the prevalence of elevated transaminase levels based on COVID-19 severity. In Mao et al., odds ratios were used to describe the probability of abnormal liver chemistry, including increased ALT and AST levels according to disease severity, and found a higher risk of abnormal liver chemistry in patients with severe COVID-19 [11]. In the present study, we provided the exact magnitude of the prevalence of elevated ALT and the prevalence of elevated AST in severe and non-severe COVID-19 patients, which were 30\% versus $15 \%$, and $44 \%$ versus $11 \%$, respectively. We also provided the prevalence of elevated ALT and the prevalence of elevated AST in critically ill cases as well as fatal cases, and found the differences were not significant between the two subgroups. Because the overall prevalence of elevated ALT and AST is influenced by the proportion of studies enrolling patients with severe COVID-19 in meta-analyses, we think that epidemiologic data are more valuable when reported for different subpopulations stratified by severity of COVID-19.

The prevalence of elevated transaminase levels in paediatric patients with COVID-19 showed that elevated ALT in this subpopulation were significantly lower than those of adult patients, which is consistent with a previous study [11]. However, unlike the study conducted by Mao et al. [11], the difference in AST was not significant. We further compared the prevalence of elevated ALT and the prevalence of elevated AST in paediatric patients compared to adults with severe and non-severe COVID-19. The differences seemed to be driven by adult patients with severe COVID-19. Therefore, we suppose that no significant difference in the elevated transaminase exists between paediatric and adults with non-severe COVID-19, because most paediatric patients have clinically milder symptoms and favourable prognosis $[95,96]$. However, due to inadequate studies with adult patients with non-severe COVID-19, the corollary needs to be further confirmed.

Among the studies included in the present meta-analysis, three discussed the association between elevated transaminase and the prognosis of patients, and the results were inconsistent partially because of different analysis methods and outcome indicators $[33,72,76]$. Recently, one study evaluated the association of mild vs severe liver injury with 
mortality in COVID-19 patients and found that severe liver injury in this cohort was associated with multiple-organ failure and mortality. Whereas mild liver injury was not related to poor outcomes [97]. Hence, although our meta-analysis showed that the prevalence of liver injury defined by strict criteria was only $9 \%$, severe liver injury is worthy of note. More research is needed to address whether transaminase elevation or liver injury is an independent risk factor for death in COVID-19 patients.

Sensitivity analyses were performed to verify the robustness of the results regarding the overall prevalence of increased aminotransferase and liver injury. As a result, the adjusted point estimates were remarkably different from the unadjusted ones. The adjusted prevalence of increased ALT, increased AST, and liver injury in the COVID-19 patients were all decreased, which indicates that the overall prevalence of hypertransaminemia and liver injury in patients with COVID-19 might be overestimated.

Compared to previous studies, there are several strengths in our study. First, we show evidence that the definition of liver injury might have a significant impact on the reported prevalence of liver injury in COVID-19 patients. Second, we found that the liver injury defined with strict criteria might be not common. Third, a sensitivity analysis was performed to estimate the robustness of the results obtained by the trim and fill method, which showed that both hypertransaminemia and liver injury may be overestimated in COVID-19 patients.

This meta-analysis also had some limitations. First, as the research on COVID-19 progresses rapidly, the most recently published literature could not be included. Second, there was large heterogeneity among the studies where the normal range of aminotransferases and the definition of liver injury were not consistent. Third, significant publication bias in the meta-analysis was found. Finally, most studies had a small sample size.

In conclusion, our study found the definition of liver injury might have a significant impact on the reported prevalence in COVID-19 patients and provided the exact magnitude of prevalence of elevated ALT and AST in non-severe and severe COVID-19 patients, as well as critically ill and fatal cases. Our study showed that only a small fraction of COVID-19 patients had clinically significant liver injury defined by strict criteria. Sensitivity analyses indicated that the overall prevalence of hypertransaminemia and liver injury might be overestimated. However, in patients with severe COVID-19, more attention should be paid to liver function tests.

\section{Supplementary Material}

Supplementary figures and tables.

http://www.medsci.org/v18p1285s1.pdf

\section{Author Contributions}

Yongxing $\mathrm{Xu}$, Jianwen $\mathrm{Gu}$, and Pengfei Liu conceived the study. Jianwen $\mathrm{Gu}$ supervised the whole study and manuscript editing. Danyang Gao, Yitian Yang, and Gang Li drafted the manuscript. Gang Li, Pengfei Liu, and Yongxing Xu extracted the data. Yongxing $\mathrm{Xu}$, Gang Li, Pengfei Liu and Yitian Yang analysed the data. Yitian Yang, Gang Li, Danyang Gao, Yongxing $\mathrm{Xu}$, and Pengfei Liu contributed to the materials/analysis tools. All authors read and approved the final manuscript.

\section{Competing Interests}

The authors have declared that no competing interest exists.

\section{References}

1. Huang C, Wang Y, Li X, Ren L, Zhao J, Hu Y, et al. Clinical features of patients infected with 2019 novel coronavirus in Wuhan, China. The Lancet. 2020; 395: 497-506.

2. Sahu KK, Kumar R. Current perspective on pandemic of COVID-19 in the United States. J Family Med Prim Care. 2020; 9: 1784-91.

3. Alwan NA, Burgess RA, Ashworth S, Beale R, Bhadelia N, Bogaert D, et al. Scientific consensus on the COVID-19 pandemic: we need to act now. Lancet. 2020; 396: E71-E2.

4. Chen N, Zhou M, Dong X, Qu J, Gong F, Han Y, et al. Epidemiological and clinical characteristics of 99 cases of 2019 novel coronavirus pneumonia in Wuhan, China: a descriptive study. The Lancet. 2020; 395: 507-13.

5. To KK, Tsang OT, Leung WS, Tam AR, Wu TC, Lung DC, et al. Temporal profiles of viral load in posterior oropharyngeal saliva samples and serum antibody responses during infection by SARS-CoV-2: an observational cohort study. The Lancet Infectious diseases. 2020.

6. Qiu H, Wu J, Hong L, Luo Y, Song Q, Chen D. Clinical and epidemiological features of 36 children with coronavirus disease 2019 (COVID-19) in Zhejiang, China: an observational cohort study. The Lancet Infectious diseases. 2020.

7. Parohan M, Yaghoubi S, Seraj A. Liver injury is associated with severe Coronavirus disease 2019 (COVID-19) infection: a systematic review and meta-analysis of retrospective studies. Hepatology research: the official journal of the Japan Society of Hepatology. 2020.

8. Youssef M, Hussein M, Attia AS, Elshazli R, Omar M, Zora G, et al. COVID-19 and Liver Dysfunction: a systematic review and meta-analysis of retrospective studies. Journal of medical virology. 2020.

9. Li G, Xu Y, Yang YT, Liu PF. Prevalence of acute liver injury and hypertransaminemia in patients with COVID-19: a protocol for a systematic review. BMJ Open. 2020; 10: e040517.

10. Sultan S, Altayar O, Siddique SM, Davitkov P, Feuerstein JD, Lim JK, et al. AGA Institute Rapid Review of the GI and Liver Manifestations of COVID-19, Meta-Analysis of International Data, and Recommendations for the Consultative Management of Patients with COVID-19. Gastroenterology. 2020.

11. Mao R, Qiu Y, He JS, Tan JY, Li XH, Liang J, et al. Manifestations and prognosis of gastrointestinal and liver involvement in patients with COVID-19: a systematic review and meta-analysis. The lancet Gastroenterology \& hepatology. 2020.

12. Khan S, Jun L, Nawsherwan, Siddique R, Li Y, Han G, et al. Association of COVID-19 infection with pregnancy outcomes in healthcare workers and general women. Clinical microbiology and infection : the official publication of the European Society of Clinical Microbiology and Infectious Diseases. 2020.

13. Bangash MN, Patel J, Parekh D. COVID-19 and the liver: little cause for concern. The lancet Gastroenterology \& hepatology. 2020; 5: 529-30.

14. Arentz M, Yim E, Klaff L, Lokhandwala S, Riedo FX, Chong M, et al. Characteristics and Outcomes of 21 Critically Ill Patients with COVID-19 in Washington State. JAMA - Journal of the American Medical Association. 2020.

15. Wang $Y$, Lu $X$, Chen $H$, Chen $T$, Su N, Huang $F$, et al Clinical Course and Outcomes of 344 Intensive Care Patients with COVID-19. American journal of respiratory and critical care medicine. 2020. 
16. Moher D, Liberati A, Tetzlaff J, Altman DG. Preferred reporting items for systematic reviews and meta-analyses: the PRISMA statement. Annals of internal medicine. 2009; 151: 264-9, w64

17. Munn Z, Moola S, Lisy K, Riitano D, Tufanaru C. Methodological guidance for systematic reviews of observational epidemiological studies reporting prevalence and cumulative incidence data. International journal of evidence-based healthcare. 2015; 13: 147-53.

18. Aithal GP, Watkins PB, Andrade RJ, Larrey D, Molokhia M, Takikawa H, et al. Case definition and phenotype standardization in drug-induced liver injury. Clinical pharmacology and therapeutics. 2011; 89: 806-15.

19. Robles-Diaz M, Lucena MI, Kaplowitz N, Stephens C, Medina-Cáliz I, González-Jimenez A, et al. Use of Hy's law and a new composite algorithm to predict acute liver failure in patients with drug-induced liver injury. Gastroenterology. 2014; 147: 109-18.e5.

20. Vasikasin V, Rojdumrongrattana T, Chuerboonchai W, Siriwiwattana T, Thongtaeparak W, Niyasom S, et al. Effect of standard dose paracetamol versus placebo as antipyretic therapy on liver injury in adult dengue infection: a multicentre randomised controlled trial. The Lancet Global health. 2019; 7: e664-e70.

21. DerSimonian R, Laird N. Meta-analysis in clinical trials. Controlled clinical trials. 1986; $7: 177-88$

22. Higgins JP, Thompson SG, Deeks JJ, Altman DG. Measuring inconsistency in meta-analyses. BMJ (Clinical research ed). 2003; 327: 557-60.

23. Duval S, Tweedie R. Trim and fill: A simple funnel-plot-based method of testing and adjusting for publication bias in meta-analysis. Biometrics. 2000; 56: 455-63.

24. Tang KL, Caffrey NP, Nóbrega DB, Cork SC, Ronksley PE, Barkema HW, et al. Restricting the use of antibiotics in food-producing animals and its associations with antibiotic resistance in food-producing animals and human beings: a systematic review and meta-analysis. The Lancet Planetary health. 2017; 1: e316-e27.

25. Begg CB, Mazumdar M. Operating characteristics of a rank correlation test for publication bias. Biometrics. 1994; 50: 1088-101.

26. Egger M, Davey Smith G, Schneider M, Minder C. Bias in meta-analysis detected by a simple, graphical test. BMJ (Clinical research ed). 1997; 315: 629-34.

27. Bhatraju PK, Ghassemieh BJ, Nichols M, Kim R, Jerome KR, Nalla AK, et al. Covid-19 in Critically Ill Patients in the Seattle Region - Case Series. N Engl J Med. 2020.

28. Cai J, Xu J, Lin D, Yang Z, Xu L, Qu Z, et al. A Case Series of children with 2019 novel coronavirus infection: clinical and epidemiological features. Clinical infectious diseases : an official publication of the Infectious Diseases Society of America. 2020.

29. Cai Q, Huang D, Ou P, Yu H, Zhu Z, Xia Z, et al. COVID-19 in a Designated Infectious Diseases Hospital Outside Hubei Province, China. Allergy. 2020.

30. Cao B, Wang Y, Wen D, Liu W, Wang J, Fan G, et al. A Trial of Lopinavir-Ritonavir in Adults Hospitalized with Severe Covid-19. The New England journal of medicine. 2020.

31. Cao J, Tu WJ, Cheng W, Yu L, Liu YK, Hu X, et al. Clinical Features and Short-term Outcomes of 102 Patients with Corona Virus Disease 2019 in Wuhan, China. Clinical infectious diseases : an official publication of the Infectious Diseases Society of America. 2020.

32. Chen L, Liu HG, Liu W, Liu J, Liu K, Shang J, et al. [Analysis of clinical features of 29 patients with 2019 novel coronavirus pneumonia]. Zhonghua jie he he hu xi za zhi = Zhonghua jiehe he huxi zazhi = Chinese journal of tuberculosis and respiratory diseases. 2020; 43: 203-8.

33. Chen T, Dai Z, Mo P, Li X, Ma Z, Song S, et al. Clinical characteristics and outcomes of older patients with coronavirus disease 2019 (COVID-19) in Wuhan, China (2019): a single-centered, retrospective study. The journals of gerontology Series A, Biological sciences and medical sciences. 2020.

34. Chen X, Yang Y, Huang M, Liu L, Zhang X, Xu J, et al. Differences between COVID-19 and suspected then confirmed SARS-CoV-2-negative pneumonia: a retrospective study from a single center. Journal of medical virology. 2020.

35. Du RH, Liu LM, Yin W, Wang W, Guan LL, Yuan ML, et al. Hospitalization and Critical Care of 109 Decedents with COVID-19 Pneumonia in Wuhan, China. Annals of the American Thoracic Society. 2020.

36. Du Y, Tu L, Zhu P, Mu M, Wang R, Yang P, et al. Clinical Features of 85 Fatal Cases of COVID-19 from Wuhan: A Retrospective Observational Study. American journal of respiratory and critical care medicine. 2020.

37. Guan WJ, Ni ZY, Hu Y, Liang WH, Ou CQ, He JX, et al. Clinical Characteristics of Coronavirus Disease 2019 in China. The New England journal of medicine. 2020.

38. Guo T, Fan Y, Chen M, Wu X, Zhang L, He T, et al. Cardiovascular Implications of Fatal Outcomes of Patients with Coronavirus Disease 2019 (COVID-19). JAMA Cardiology. 2020.

39. Han X, Cao Y, Jiang N, Chen $Y$, Alwalid O, Zhang X, et al. Novel Coronavirus Pneumonia (COVID-19) Progression Course in 17 Discharged Patients: Comparison of Clinical and Thin-Section CT Features During Recovery. Clinical infectious diseases : an official publication of the Infectious Diseases Society of America. 2020.

40. Han YN, Feng ZW, Sun LN, Ren XX, Wang H, Xue YM, et al. A comparative-descriptive analysis of clinical characteristics in 2019-Coronavirus-infected children and adults. Journal of medical virology. 2020.
41. He XW, Lai JS, Cheng J, Wang MW, Liu YJ, Xiao ZC, et al. Impact of complicated myocardial injury on the clinical outcome of severe or critically ill COVID-19 patients. Zhonghua xin xue guan bing za zhi. 2020; 48: E011.

42. Hu Z, Song C, Xu C, Jin G, Chen Y, Xu X, et al. Clinical characteristics of 24 asymptomatic infections with COVID-19 screened among close contacts in Nanjing, China. Sci China Life Sci. 2020; 63: 706-11.

43. Huang $\mathrm{Y}, \mathrm{Tu} \mathrm{M}$, Wang $\mathrm{S}$, Chen $\mathrm{S}$, Zhou W, Chen $\mathrm{D}$, et al. Clinical characteristics of laboratory confirmed positive cases of SARS-CoV-2 infection in Wuhan, China: A retrospective single center analysis. Travel Medicine and Infectious Disease. 2020

44. Ji D, Qin E, Xu J, Zhang D, Cheng G, Wang Y, et al. Implication of non-alcoholic fatty liver diseases (NAFLD) in patients with COVID-19: a preliminary analysis. Journal of hepatology. 2020.

45. Kim ES, Chin BS, Kang CK, Kim NJ, Kang YM, Choi JP, et al. Clinical Course and Outcomes of Patients with Severe Acute Respiratory Syndrome Coronavirus 2 Infection: a Preliminary Report of the First 28 Patients from the Korean Cohort Study on COVID-19. Journal of Korean medical science. 2020; 35: e142.

46. Lei Z, Cao H, Jie Y, Huang Z, Guo X, Chen J, et al. A cross-sectional comparison of epidemiological and clinical features of patients with coronavirus disease (COVID-19) in Wuhan and outside Wuhan, China. Travel medicine and infectious disease. 2020: 101664.

47. Li YK, Peng S, Li LQ, Wang Q, Ping W, Zhang N, et al. Clinical and Transmission Characteristics of Covid-19 - A Retrospective Study of 25 Cases from a Single Thoracic Surgery Department. Current medical science. 2020.

48. Lian J, Jin X, Hao S, Cai H, Zhang S, Zheng L, et al. Analysis of Epidemiological and Clinical features in older patients with Corona Virus Disease 2019 (COVID-19) out of Wuhan. Clinical infectious diseases : an official publication of the Infectious Diseases Society of America. 2020.

49. Lin L, Jiang X, Zhang Z, Huang S, Zhang Z, Fang Z, et al. Gastrointestinal symptoms of 95 cases with SARS-CoV-2 infection. Gut. 2020

50. Liu C, Jiang ZC, Shao CX, Zhang HG, Yue HM, Chen ZH, et al. Preliminary study of the relationship between novel coronavirus pneumonia and liver function damage: a multicenter study. Zhonghua gan zang bing za zhi $=$ Zhonghua ganzangbing zazhi $=$ Chinese journal of hepatology. 2020; 28: 148-52.

51. Liu M, He P, Liu HG, Wang XJ, Li FJ, Chen S, et al. [Clinical characteristics of 30 medical workers infected with new coronavirus pneumonia]. Zhonghua jie he he hu xi za zhi = Zhonghua jiehe he huxi zazhi = Chinese journal of tuberculosis and respiratory diseases. 2020; 43: 209-14.

52. Lo IL, Lio CF, Cheong HH, Lei CI, Cheong TH, Zhong $\mathrm{X}$, et al. Evaluation of SARS-CoV-2 RNA shedding in clinical specimens and clinical characteristics of 10 patients with COVID-19 in Macau. International journal of biological sciences. 2020; 16: 1698-707.

53. Lu X, Zhang L, Du H, Zhang J, Li YY, Qu J, et al. SARS-CoV-2 Infection in Children. N Engl J Med. 2020; 382: 1663-5.

54. Mi B, Chen L, Xiong Y, Xue H, Zhou W, Liu G. Characteristics and Early Prognosis of COVID-19 Infection in Fracture Patients. The Journal of bone and joint surgery American volume. 2020.

55. Qian GQ, Yang NB, Ding F, Ma AHY, Wang ZY, Shen YF, et al. Epidemiologic and Clinical Characteristics of 91 Hospitalized Patients with COVID-19 in Zhejiang, China: A retrospective, multi-centre case series. QJM : monthly journal of the Association of Physicians. 2020.

56. Qian ZP, Mei X, Zhang YY, Zou Y, Zhang ZG, Zhu H, et al. [Analysis of baseline liver biochemical parameters in 324 cases with novel coronavirus pneumonia in Shanghai area]. Zhonghua gan zang bing za zhi = Zhonghua ganzangbing zazhi = Chinese journal of hepatology. 2020; 28: E005.

57. Qiu L, Liu X, Xiao M, Xie J, Cao W, Liu Z, et al. SARS-CoV-2 is not detectable in the vaginal fluid of women with severe COVID-19 infection. Clinical infectious diseases : an official publication of the Infectious Diseases Society of America. 2020

58. Shi H, Han X, Jiang N, Cao Y, Alwalid O, Gu J, et al. Radiological findings from 81 patients with COVID-19 pneumonia in Wuhan, China: a descriptive study. The Lancet Infectious Diseases. 2020; 20: 425-34.

59. Tu WJ, Cao J, Yu L, Hu X, Liu Q. Clinicolaboratory study of 25 fatal cases of COVID-19 in Wuhan. Intensive care medicine. 2020.

60. Wan S, Xiang Y, Fang W, Zheng Y, Li B, Hu Y, et al. Clinical features and treatment of COVID-19 patients in northeast Chongqing. Journal of Medical Virology. 2020.

61. Wang D, Ju XL, Xie F, Lu Y, Li FY, Huang HH, et al. Clinical analysis of 31 cases of 2019 novel coronavirus infection in children from six provinces (autonomous region) of northern China. Zhonghua er ke za zhi $=$ Chinese journal of pediatrics. 2020; 58: E011.

62. Wang F, Wang H, Fan J, Zhang Y, Wang H, Zhao Q. Pancreatic Injury Patterns in Patients With Coronavirus Disease 19 Pneumonia. Gastroenterology. 2020.

63. Wang L, Gao YH, Lou LL, Zhang GJ. The clinical dynamics of 18 cases of COVID-19 outside of Wuhan, China. The European respiratory journal. 2020.

64. Wang Z, Yang B, Li Q, Wen L, Zhang R. Clinical Features of 69 Cases with Coronavirus Disease 2019 in Wuhan, China. Clinical infectious diseases : an official publication of the Infectious Diseases Society of America. 2020.

65. Wu J, Liu J, Zhao X, Liu C, Wang W, Wang D, et al. Clinical Characteristics of Imported Cases of COVID-19 in Jiangsu Province: A Multicenter Descriptive Study. Clinical infectious diseases : an official publication of the Infectious Diseases Society of America. 2020. 
66. Xu XW, Wu XX, Jiang XG, Xu KJ, Ying LJ, Ma CL, et al. Clinical findings in a group of patients infected with the 2019 novel coronavirus (SARS-Cov-2) outside of Wuhan, China: Retrospective case series. The BMJ. 2020; 368.

67. Xu Y, Li X, Zhu B, Liang H, Fang C, Gong Y, et al. Characteristics of pediatric SARS-CoV-2 infection and potential evidence for persistent fecal viral shedding. Nature Medicine. 2020.

68. Yang W, Cao O, Oin L, Wang X, Cheng Z, Pan A, et al. Clinical characteristics and imaging manifestations of the 2019 novel coronavirus disease (COVID-19):A multi-center study in Wenzhou city, Zhejiang, China. Journal of Infection. 2020; 80: 388-93.

69. Yang X, Yu Y, Xu J, Shu H, Xia J, Liu H, et al. Clinical course and outcomes of critically ill patients with SARS-CoV-2 pneumonia in Wuhan, China: a single-centered, retrospective, observational study. Lancet Respir Med. 2020; 8: 475-81.

70. Yao N, Wang SN, Lian JQ, Sun YT, Zhang GF, Kang WZ, et al. Clinical characteristics and influencing factors of patients with novel coronavirus pneumonia combined with liver injury in Shaanxi region. Zhonghua gan zang bing za zhi $=$ Zhonghua ganzangbing zazhi $=$ Chinese journal of hepatology. 2020; 28: E003.

71. Zha L, Li S, Pan L, Tefsen B, Li Y, French N, et al. Corticosteroid treatment of patients with coronavirus disease 2019 (COVID-19). The Medical journal of Australia. 2020.

72. Zhang G, Zhang J, Wang B, Zhu X, Wang Q, Qiu S. Analysis of clinical characteristics and laboratory findings of 95 cases of 2019 novel coronavirus pneumonia in Wuhan, China: A retrospective analysis. Respiratory Research. 2020; 21.

73. Zhang Y, Zheng L, Liu L, Zhao M, Xiao J, Zhao Q. Liver impairment in COVID-19 patients: a retrospective analysis of 115 cases from a single center in Wuhan city, China. Liver international : official journal of the International Association for the Study of the Liver. 2020.

74. Zhao D, Yao F, Wang L, Zheng L, Gao Y, Ye J, et al. A comparative study on the clinical features of COVID-19 pneumonia to other pneumonias. Clinical infectious diseases : an official publication of the Infectious Diseases Society of America. 2020.

75. Zheng F, Tang W, Li H, Huang YX, Xie YL, Zhou ZG. Clinical characteristics of 161 cases of corona virus disease 2019 (COVID-19) in Changsha. European review for medical and pharmacological sciences. 2020; 24: 3404-10.

76. Zhou F, Yu T, Du R, Fan G, Liu Y, Liu Z, et al. Clinical course and risk factors for mortality of adult inpatients with COVID-19 in Wuhan, China: a retrospective cohort study. The Lancet. 2020.

77. Zhu L, Wang J, Huang R, Liu L, Zhao H, Wu C, et al. Clinical characteristics of a case series of children with coronavirus disease 2019. Pediatric pulmonology. 2020.

78. Chen T, Wu D, Chen H, Yan W, Yang D, Chen G, et al. Clinical characteristics of 113 deceased patients with coronavirus disease 2019: retrospective study. BMJ (Clinical research ed). 2020; 368: m1091.

79. Chen G, Wu D, Guo W, Cao Y, Huang D, Wang H, et al. Clinical and immunologic features in severe and moderate Coronavirus Disease 2019. The Journal of clinical investigation. 2020.

80. Hamming I, Timens W, Bulthuis ML, Lely AT, Navis G, van Goor H. Tissue distribution of ACE2 protein, the functional receptor for SARS coronavirus. A first step in understanding SARS pathogenesis. The Journal of pathology. 2004; 203: 631-7.

81. Li G, Fan Y, Lai Y, Han T, Li Z, Zhou P, et al. Coronavirus infections and immune responses. Journal of medical virology. 2020; 92: 424-32.

82. Lelubre C, Vincent JL. Mechanisms and treatment of organ failure in sepsis. Nature reviews Nephrology. 2018; 14: 417-27.

83. Katarey D, Verma S. Drug-induced liver injury. Clinical medicine (London, England). 2016; 16: s104-s9.

84. Zhang C, Shi L, Wang FS. Liver injury in COVID-19: management and challenges. Lancet Gastroenterol Hepatol. 2020; 5: 428-30.

85. Pagnesi M, Baldetti L, Beneduce A, Calvo F, Gramegna M, Pazzanese V, et al. Pulmonary hypertension and right ventricular involvement in hospitalised patients with COVID-19. Heart. 2020; 106: 1324-31.

86. Zeng JH, Wu WB, Ou JX, Wang $\mathrm{Y}$, Dong $\mathrm{CF}$, Luo $\mathrm{YF}$, et al. Cardiac manifestations of COVID-19 in Shenzhen, China. Infection. 2020.

87. Farha S. COVID-19 and pulmonary hypertension. Cleve Clin J Med. 2020.

88. Cagli K, Basar FN, Tok D, Turak O, Basar O. How to interpret liver function tests in heart failure patients? Turk J Gastroenterol. 2015; 26: 197-203.

89. Henry BM, de Oliveira MHS, Benoit S, Plebani M, Lippi G. Hematologic, biochemical and immune biomarker abnormalities associated with severe illness and mortality in coronavirus disease 2019 (COVID-19): a meta-analysis. Clinical chemistry and laboratory medicine. 2020.

90. Kukla M, Skonieczna-Żydecka K, Kotfis K, Maciejewska D, Łoniewski I, Lara LF, et al. COVID-19, MERS and SARS with Concomitant Liver InjurySystematic Review of the Existing Literature. Journal of clinical medicine. 2020; 9.

91. Kunutsor SK, Laukkanen JA. Markers of liver injury and clinical outcomes in COVID-19 patients: A systematic review and meta-analysis. The Journal of infection. 2020.

92. Wang H, Qiu P, Liu J, Wang F, Zhao Q. The liver injury and gastrointestinal symptoms in patients with Coronavirus Disease 19: A systematic review and meta-analysis. Clin Res Hepatol Gastroenterol. 2020.
93. Lv XH, Yang JL, Deng K. Letter to the Editors: COVID-19-Related Liver Injury: The Interpretation for Aspartate Aminotransferase Needs to Be Cautious. Hepatology. 2020.

94. $\mathrm{Xu} \mathrm{Y,} \mathrm{Liu} \mathrm{P,} \mathrm{Gu} \mathrm{J.} \mathrm{Gastrointestinal} \mathrm{and} \mathrm{liver} \mathrm{involvement} \mathrm{in} \mathrm{patients} \mathrm{with}$ COVID-19. Lancet Gastroenterol Hepatol. 2020; 5: 798-9.

95. Wang Z, Zhou Q, Wang C, Shi Q, Lu S, Ma Y, et al. Clinical characteristics of children with COVID-19: a rapid review and meta-analysis. Annals of translational medicine. 2020; 8: 620 .

96. Shekerdemian LS, Mahmood NR, Wolfe KK, Riggs BJ, Ross CE, McKiernan CA, et al. Characteristics and Outcomes of Children With Coronavirus Disease 2019 (COVID-19) Infection Admitted to US and Canadian Pediatric Intensive Care Units. JAMA pediatrics. 2020.

97. Anastasiou OE, Korth J, Herbstreit F, Witzke O, Lange CM. Mild vs severe liver injury in SARS-CoV-2 infection. Dig Dis. 2020. 